\title{
Perceptions of Fetal Alcohol Spectrum Disorder (FASD) among School Superintendents in the State of Minnesota
}

\author{
Jerrod Brown ${ }^{1,2,3}{ }^{*}$, Donald Helmstetter ${ }^{1}$, Diane Harr ${ }^{1}$ and Jeff Louie ${ }^{4}$ \\ ${ }^{1}$ Concordia University, St. Paul, MN, USA \\ ${ }^{2}$ Pathways Counseling Center, St. Paul, MN, USA \\ ${ }^{3}$ The American Institute for the Advancement of Forensic Studies, St. Paul, MN, USA \\ ${ }^{4}$ University of Minnesota, Department of Pediatrics, Minneapolis, MN, USA
}

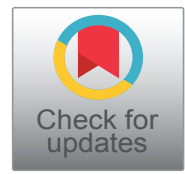

*Corresponding author: Jerrod Brown, Ph.D., MA, MS, Pathways Counseling Center, 1919 University Ave W Suite 6 St. Paul MN, 55104, St. Paul, MN, USA, E-mail: jerrod01234brown@live.com

\begin{abstract}
Children with Fetal Alcohol Spectrum Disorders (FASD) offer unique challenges in school and other settings. As such, administrators such as district superintendents, many of whom make recommendations for long-term suspensions or expulsion, need to be prepared to work with students with FASD. This requires familiarity with FASD screening, knowledge of FASD intervention and teaching strategies, and possession of a plan for referring these students for additional assistance. Unfortunately, little is known about how familiar school superintendents are with FASD. To this end, a Web-based qualitative survey concerning FASD was administered to school superintendents. This study found that superintendents vary in their (a) Ability to accurately identify signs and symptoms of FASD and (b) Awareness of unique psychoeducational impairments experienced by these individuals. Superintendents believe they would benefit from continuing education in the psychoeducational impairments of individuals diagnosed with FASD and a form or checklist to assist them in their decision-making process.
\end{abstract}

\section{Keywords}

FASD, Education, School superintendents, Special education, Training

\section{Introduction}

Afflicting 2-5\% of the U.S. population [1], Fetal Alcohol Spectrum Disorder (FASD) is not an easily recognized disorder. Caused by Prenatal Alcohol Exposure (PAE) and its associated brain damage [2], FASD can present in a variety of ways. This can include cognitive (e.g., executive control, learning, memory, attention, and impulsivity), social (e.g., communication including verbal and non-verbal language), and adaptive (e.g., decision-making, problem-solving, and ability to learn from experience) impairments [3-6]. In education settings, deficits in short- and long-term memory and the capacity to learn may be the most noticeable [7-9]. Deficits in the encoding, retrieval, and application of memory [10] can impact verbal and non-verbal learning, mathematics, and the identification of odors and colors [11,12]. Because these symptoms can vary widely as a function of the timing and amount of prenatal alcohol exposure, FASD often goes unrecognized, undiagnosed, and subsequently untreated in school children.

Diagnosis is further complicated in at least three ways. First, individuals with FASD typically do not have visible indicators of the disorder such as physical deformities. Second, many of the associated impairments can be, and often are, superficially masked by the individual. This is particularly true over short periods of time in comparison to longer ones that allow for more observation as part of a long-term relationship. For example, individuals with FASD may mask the disorder by scoring in the normal range on intelligence tests or presenting with adequate verbal intelligence. However, difficulties in global functioning tend to become apparent over longer periods of interaction $[13,14]$. Third, almost all individuals with FASD have at least one co-occurring mental disorder [12]. In fact, one study estimated that this was the case for over $90 \%$ of individuals with FASD

Citation: Brown J, Helmstetter D, Harr D, Louie J (2017) Perceptions of Fetal Alcohol Spectrum Disorder (FASD) among School Superintendents in the State of Minnesota. Int J Neurol Neurother 4:068. doi. org/10.23937/2378-3001/1410068

Received: August 18, 2017: Accepted: October 31, 2017: Published: November 02, 2017

Copyright: (C) 2017 Brown J, et al. This is an open-access article distributed under the terms of the Creative Commons Attribution License, which permits unrestricted use, distribution, and reproduction in any medium, provided the original author and source are credited. 
[15]. Common comorbid disorders include behavioral (e.g., Attention-Deficit/Hyperactivity Disorder (ADHD), conduct disorder, and oppositional defiant disorder), mood (e.g., depression and mania), substance use, trauma and Post-Traumatic Stress Disorder (PTSD), and learning disorders [2,15-18]. When not accurately identified, FASD typically goes untreated in school children, perpetuating a host of learning deficits and inappropriate behaviors that can impinge on social development.

Outside of immediate family, educators typically spend more time with the child than almost anyone else. Although educators typically have a limited recognition and understanding of FASD, teachers and other district staff are in a vital, unique, and influential position to recognize the challenging symptoms of FASD through their daily student interactions. Empowering teachers to recognize and refer students to appropriate qualified professionals significantly increases the likelihood of positives outcomes for all students with FASD. When FASD is suspected, the student should be referred to an educational assessment team for multidisciplinary planning and potential formalized assessment. Such an endeavor should incorporate information from family and teachers on the student's strengths and weaknesses. Thorough and accurate diagnosis is critical to informing the development of treatment and case management plans for the student. As always, early intervention provides students with the best chance for long-term success and the minimization of consequences across the lifespan [19-21].

The cost of FASD going unrecognized within students is not simply monetary. Students with FASD often exhibit lower rates of achievement [15] with a higher likelihood of truancy, behavioral problems, and failure to graduate relative to students without FASD [7,15,22]. Further, students with FASD can be more challenging to instruct and require increased resources in comparison to other non-FASD students. Specifically, students with FASD may respond inconsistently to traditional teaching and disciplinary methods. For example, a student with FASD may respond positively to a technique in one instance but respond negatively to the same technique in another instance. To prevent such frustrations, teachers may benefit from considering strategies that have been found successful with students who have other special needs. The success of teachers in responding to the diverse challenges of students with FASD holds promise in improving the academic success of these students $[19,20,21,23]$.

The key to improving outcomes for students with FASD may be the incorporation of a team approach. In addition to parental involvement, a network of dedicated teachers, advocates, and school administrators all have important roles to play. First, teachers will always be at the forefront of these efforts. Teachers can play an integral role in identifying students who may have FASD, ensuring referral for proper assessment, and finding ways to improve the effectiveness of their learning program. Second, once identified as a student with FASD, the district should identify an advocate for the student. Ideally, this should be an education professional that has made a strong connection with the student. This advocate has the task of ensuring that teachers are aware of the student's strengths and weaknesses throughout transitions in the educational process. Third, school district administrators can help ensure that staff is familiar with the best available strategies and techniques for educating this challenging population. Further, these administrators have the opportunity to help address behavioral problems in ways that limit the development of long-term consequences such as expulsion. Throughout this process, this team should involve mental health and social services professionals when appropriate to maximize the effectiveness of interventions.

Superintendents, and their district staff, receive limited education and training about FASD and its impacts within the education system. At present, research is inconclusive on the efficacy and Return on Investment (ROI) that results from providing treatment to these students. It is believed that when this student population is supported with adequate resources their long-term outcomes like school performance, social functioning, and problematic behaviors will dramatically improve. District staff at all levels can benefit from better understanding and awareness about FASD and how it impacts the lives of those students with FASD.

\section{The present study}

The purpose of the present study is to determine how well educators understand FASD. Because top-level administrators are often tasked with important decisions including long-term suspension and expulsion, school superintendents are the focal point of this study. This is an understudied area, as the authors were unable to identify any existing publications worldwide that explore this topic among school district superintendents. The current study focused specifically on the state of Minnesota school district superintendents.

The authors hypothesized the following:

1. School superintendents, while possibly having a basic understanding of FASD, will not have an advanced understanding of the specific characteristics of FASD.

2. School superintendents have not received training on neurobehavioral impairments as they relate to FASD.

3. School superintendents believe that he or she would benefit from having the findings of an FASD screening tool as part of their daily practice.

\section{Method}

\section{Procedure}

The Perceptions of Fetal Alcohol Spectrum Disorders (FASD) among School Superintendents in the State of Minnesota was created as an online Google Sheets form. Responses were collected in a Google Sheets document and analyzed using SPSS 22 [24]. The survey was distrib- 
uted electronically to all superintendents in Minnesota ( $\mathbf{N}=\mathbf{3 2 5}$ ) by the Minnesota Association of School Administrators (MASA). After the initial recruitment email, weekly reminders were sent for a one-month period to prompt superintendents to participate in the study. Informed consent was assumed by completion, or partial completion of the survey. Study was approved by the Institutional Review Board, University of Minnesota.

\section{Measure}

The survey consisted of 13 closed-ended response questions and 1 open-ended comments question regarding participant assessments of FASD. The survey consists of 14 items, with 10 being relevant to FASD and 4 demographic questions. Demographic variables collected were: 1) Years since obtaining Minnesota Administrative License, 2) Gender, 3) MASA Region, 4) Comments. Frequency distributions were calculated for the nominal answers provided.

\section{Questions}

1. What percentage of individuals diagnosed with Fetal Alcohol Spectrum Disorder have facial abnormalities such as wide set eyes, thin upper lip, smooth philtrum, upturned nose, epicanthal folds, and small head size?

2. What percentage of individuals diagnosed with Fetal Alcohol Spectrum Disorder become involved in the criminal justice system?

3. Have you ever received training on the neurobehavioral impairments experienced by individuals diagnosed with Fetal Alcohol Spectrum Disorder?

4. How recently was this training received?

5. What do you recall from this training as to the educational developmental impairments experienced by individuals diagnosed with Fetal Alcohol Spectrum Disorder (FASD)?

6. What do you recall from this training as to the behavioral developmental impairments experienced by individuals diagnosed with Fetal Alcohol Spectrum Disorder (FASD)?

7. In your experience as a school administrator, has the behavior of a student identified with Fetal Alcohol Spectrum Disorder (FASD) been as significant as to necessitate your involvement?

8. Are you aware of any administrator in your system who knows as much or more than you do about Fetal Alcohol Spectrum Disorder (FASD)?

9. Would you likely benefit from a continuing education course on the neurobehavioral impairments of individuals diagnosed with Fetal Alcohol Spectrum Disorder (FASD)?

10. Would you likely benefit from being provided the findings of a screening tool for Fetal Alcohol Spec-

\section{Participant Gender Counts}

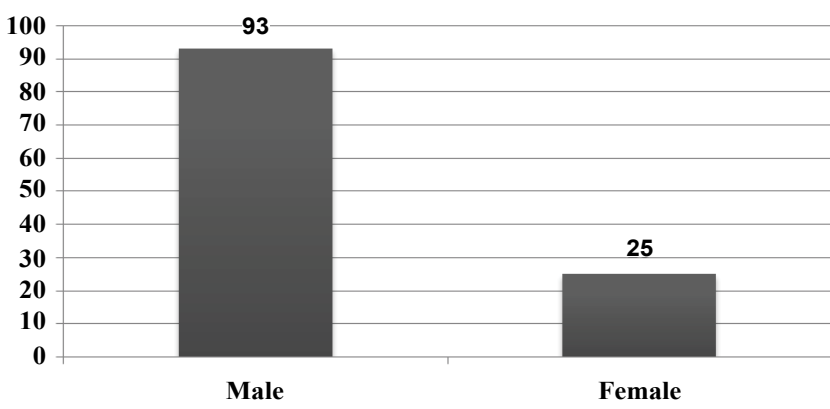

Figure 1: Participant gender counts.

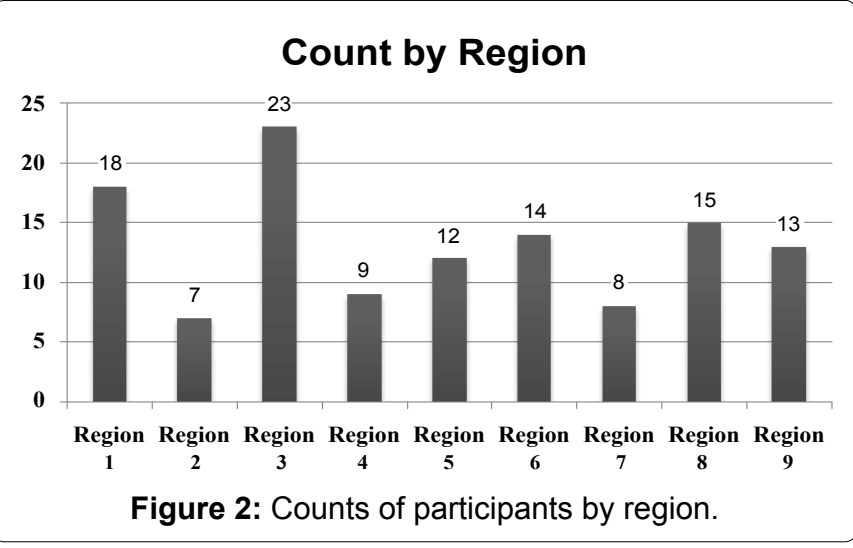

trum Disorder (FASD) in your daily practice?

11.Years since obtaining $\mathrm{MN}$ Administrative license (years)?

12. What is your gender?

13.What is your MASA region?

14.Additional Comments?

\section{Results}

\section{Respondents}

Of the 325 online survey requests sent, $37 \%(n=120)$ responded with at least a partially completed submission. Of respondents who completed the gender variable $(n=118), 21 \%$ were female $(n=25)$ and $79 \%$ were male $(n=93)$ as shown below in Figure 1 .

Nine educational service regions were established in 1976 by the Minnesota Legislature to provide cost-effective, high quality education services and programs to public schools/districts, private schools and nonprofits. Of the 119 responses by region, region 3 had the most responses at $23(19 \%)$, followed by region 1 at $18(15 \%)$, region 8 at $15(12 \%)$, region 6 at $14(12 \%)$, region 9 at $13(11 \%)$, region 5 at $12(10 \%)$, region 4 at $9(8 \%)$, region 7 at $8(7 \%)$ and region 2 at $7(6 \%)$ as shown below in Figure 2.

Of all 120 superintendents surveyed, almost 90\% had their administrative license between 5 and 10 years ( $n=24,20 \%$ ) or more than 10 years $(n=83,69 \%$ ) as shown below in Figure 3. 


\section{Years Since Licensure}

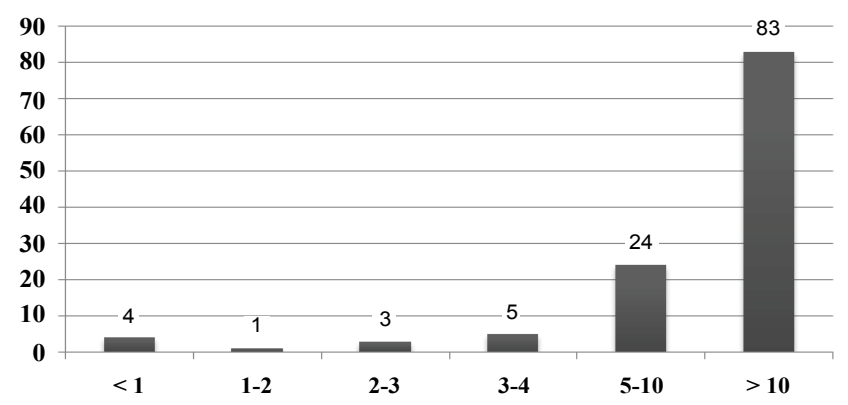

Figure 3: Years since licensure.

\section{$\%$ of FASD Individuals with physical abnormalities}

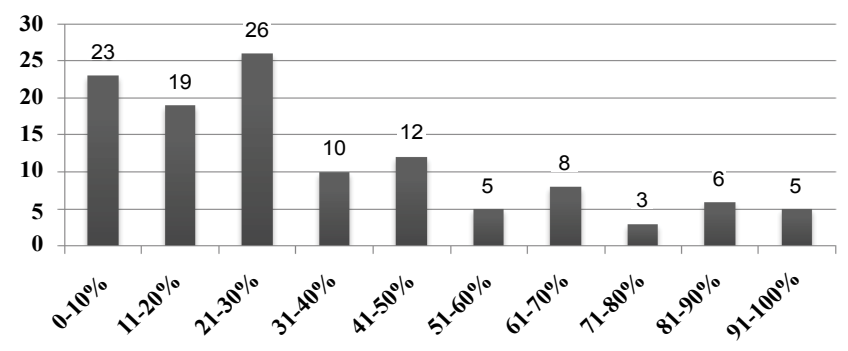

Figure 4: Percentage of individuals with FASD believed by participants to have physical abnormalities.

\section{\% FASD became crim justice involved}

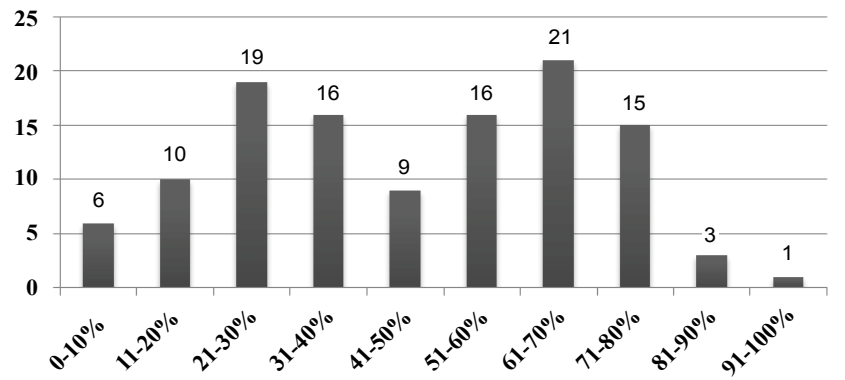

Figure 5: Counts of participants by percentage block of individuals with FASD that participants believe will come into contact with the criminal justice system at some point in their lives.

\section{FASD diagnostic knowledge}

Participants were asked a number of FASD related questions including what percentage of individuals with FASD they believed had facial abnormalities (e.g., wideset eyes, thin upper lip, smooth philtrum, upturned nose, epicanthal folds, and small head size). Of 117 respondents, the largest group believed between $21-30 \%$ ( $n=26,22 \%)$ followed by $0-10 \%(n=23,20 \%), 11-20 \%$ $(n=19,16 \%), 41-50 \%(n=12,10 \%), 31-40 \%(n=10$, $9 \%), 61-70 \%(n=89,7 \%), 81-90 \%(n=6,5 \%), 51-60 \%$ \& 91-100\% each receiving the same total (both $n=5,4 \%$ ), and $71-80 \%(n=3,3 \%)$ as shown below in Figure 4.

Participants were also asked what percentage of individuals with FASD will become involved in the criminal justice system. Of 116 respondents, the largest group
Neurobenavioral Impariments Tranining Received

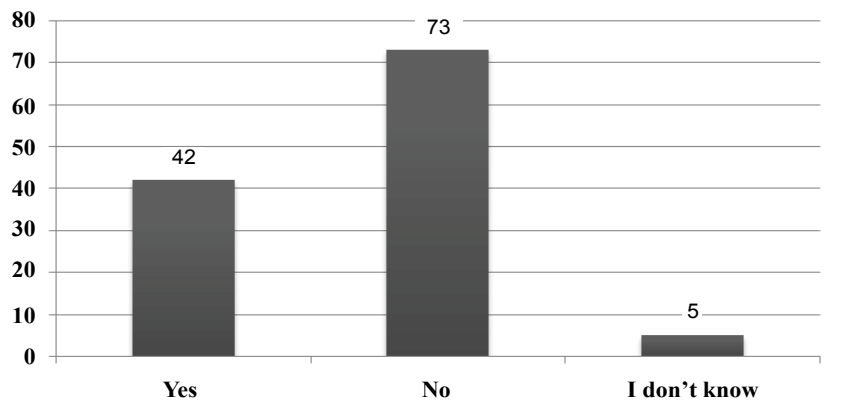

Figure 6: Count of participants regarding having received training on neurobehavioral impairments experienced by those with FASD.

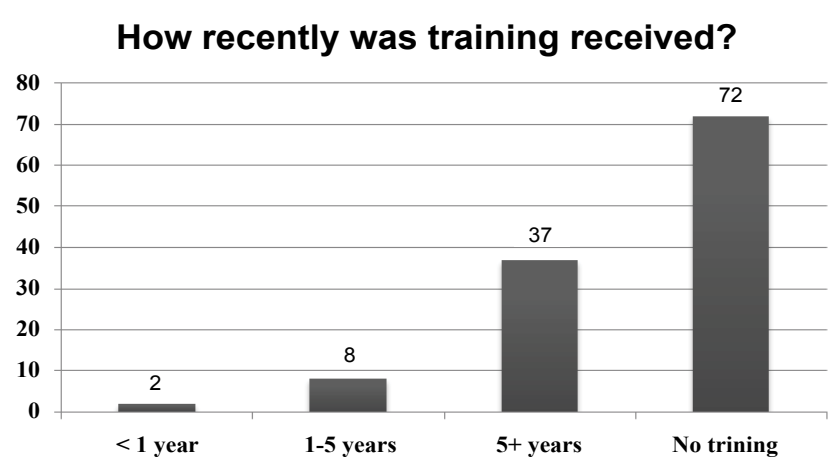

Figure 7: How long since receiving training on neurobehavioral impairment experiences of those with FASD.

believed between $61-70 \%(n=21,18 \%$ ) followed by 21 $30 \%(n=19,16 \%)$, with $31-40 \%$ and $51-60 \%$ each receiving the same total (both $n=16,14 \%), 71-80 \%(n=15$, $13 \%), 11-20 \%(n=10,9 \%), 41-50 \%(n=9,8 \%), 0-10 \%(n$ $=6,5 \%), 81-90 \%(n=3,3 \%)$, and $91-100 \%(n=1,1 \%)$ as shown below in Figure 5.

Educators are in a unique position to positively impact those students who have FASD. As they gain accurate knowledge about identification and potential implications, education professionals can also more accurately intervene on behalf of their students.

\section{Training}

Participants were also asked if they had received any training on neurobehavioral impairments experienced by individuals diagnosed with FASD. The overwhelming majority of 120 responses selected "No" $(n=73,61 \%)$, followed by "Yes" ( $n=42,35 \%)$ and "I do not know" ( $=5,4 \%$ ) as shown below in Figure 6 .

A follow-up question queried, "How recently was this training received?" Of the 119 responses, the majority replied that "I have never had training" $(n=72$, $60 \%)$, followed by over 5 years ago $(n=37,31 \%), 1-5$ years ago $(n=8,7 \%)$ and less than 1 year ago $(n=2$ and $2 \%)$ as shown below in Figure 7.

A third training related question asked, "What do you recall from this training as to the educational developmental impairments experienced by individuals diagnosed with 


\section{Educational Developmental Impairments Experienced}

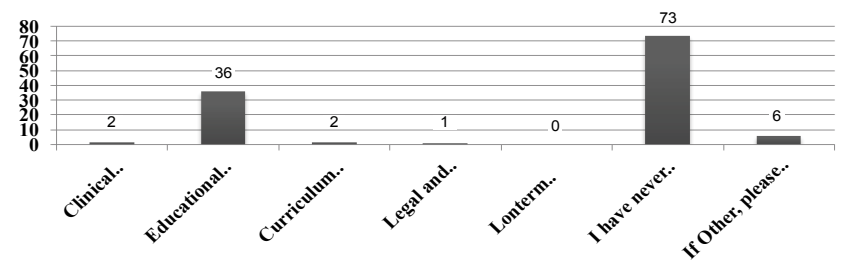

Figure 8: Count of participants regarding what they recall about the educational developmental impairments experienced by individuals with FASD.

\section{Behavioral Developmental Impairments Experienced}

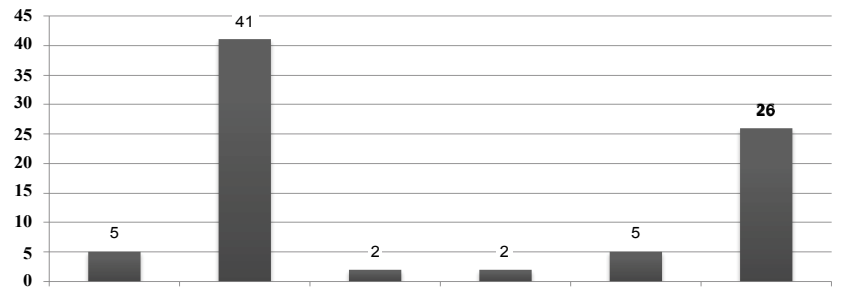

Figure 9: Count of participants regarding what they recall about the behavioral developmental impairments experienced by individuals with FASD.

FASD?" Of 120 responses, the most common response was "I have never had training" ( $n=73,61 \%)$, followed by "Educational impacts of FASD in school age children" $(n=$ $36,30 \%)$, "If Other, please provide your response" $(n=6$, $5 \%$ ), with "Clinical background of FASD" and "Curriculum regarding FASD prevention, identification, and care" each receiving the same total $(n=2,2 \%)$, "Legal and policy issues surrounding FASD" ( $n=1,1 \%)$, and "Long term planning for students with FASD" not being selected at all $(n=$ $0,0 \%)$ as shown below in Figure 8.

The final training related question was, "What do you recall from this training as to the behavioral developmental impairments experienced by individuals diagnosed with FASD?" Of 81 responses, the most common response was "Educational impacts of FASD in school age children" ( $\mathrm{n}=$ $41,51 \%)$, followed by "If Other, please provide your response" ( $n=26,32 \%)$, with "Clinical background of FASD" and "Long term planning for students with FASD" each receiving the same total $(n=5,6 \%)$, and "Curriculum regarding FASD prevention, identification, and care" and "Legal and policy issues surrounding FASD" each receiving the same total $(n=2,3 \%)$ as shown below in Figure 9.

Professional development and training opportunities which address recognition and planning for educational and behavioral needs of students with FASD should be a comprehensive part of academic institutions. Evidence-based practices should be integrated with curriculum and instruction in an effort to improve educational outcomes.

\section{Professional experience with FASD}

The last four questions of the survey dealt with professional experiences of superintendents regarding FASD. The

\section{FASD Neccessitated Involvement}

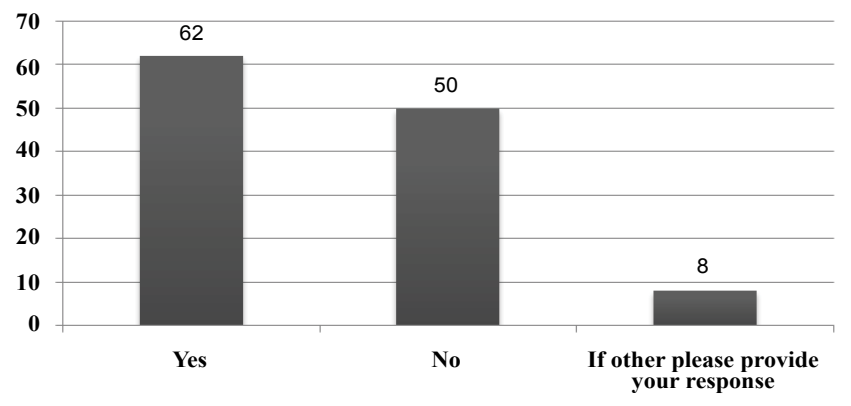

Figure 10: Count of participants that needed to get involved due to the behavior of a student with FASD.

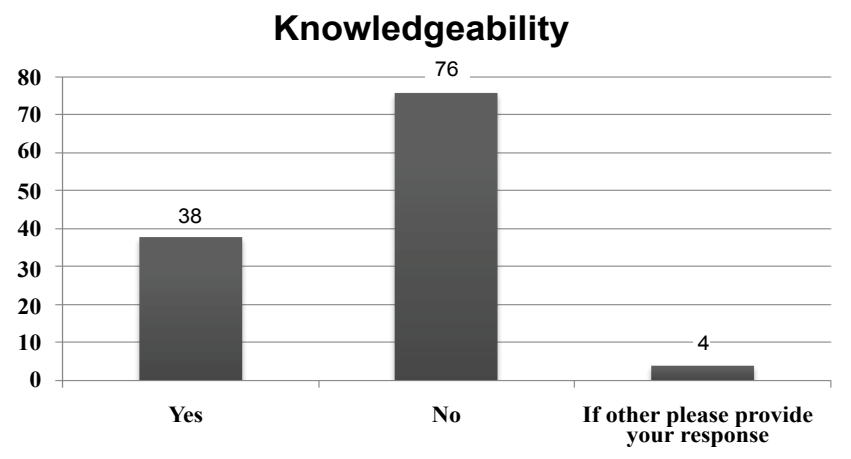

Figure 11: Count of participants regarding their knowledge ability in relation to others about FASD in their district.

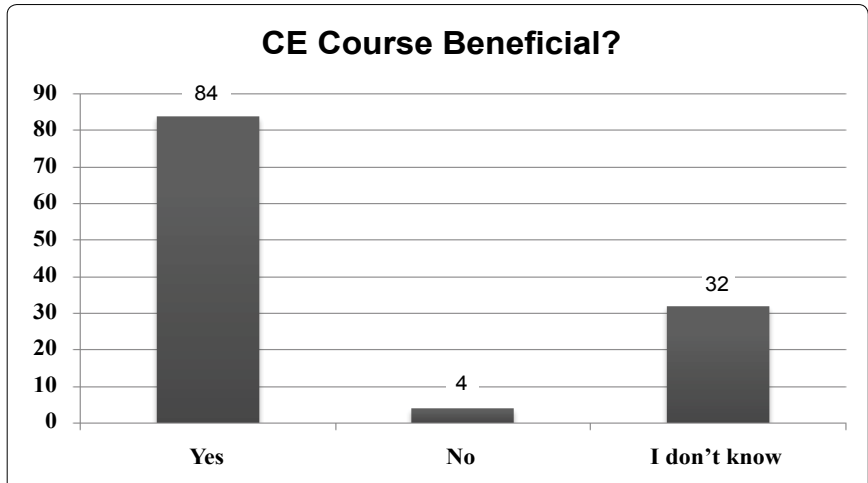

Figure 12: Would a CE course be beneficial to you?

responses to "In your experience as a school administrator has the behavior of a student identified with FASD been as significant as to necessitate your involvement?" were almost evenly split. Of 120 responses, "Yes" was selected most often ( $n=62,52 \%$ ) followed by "No" ( $n=50,42 \%)$ and "Other" ( $n=6,7 \%)$ as shown below in Figure 10.

There were 118 responses to Question 8: "Are you aware of any administrator in your system who knows as much or more than you do about FASD?" "No" ( $n=$ $76,64 \%)$ was selected most often. This was followed by "Yes" $(n=38,32 \%)$ and "Other" $(n=4,3 \%)$ as shown below in Figure 11.

There were 120 responses to Question 9: "Would you likely benefit from a continuing education course on the neurobehavioral impairments of individuals diagnosed with FASD?" The majority selected "Yes" ( $\mathrm{n}=$ $84,70 \%)$. This was followed by "I don't know" ( $n=32$, $7 \%)$ and "No" ( $n=4,3 \%)$ as shown below in Figure 12. 


\section{Screening Tool Beneficial}

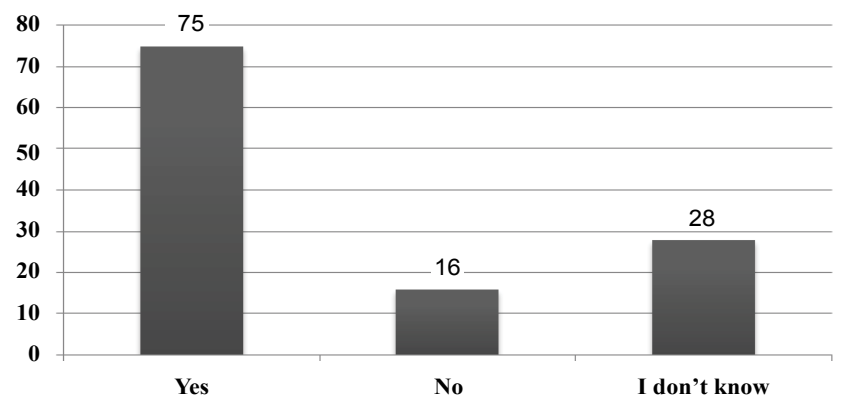

Figure 13: Would an FASD screening tool be beneficial to you?

There were 119 responses to Question 10: "Would you likely benefit from being provided the findings of a screening tool for FASD in your daily practice?" Again, the majority selected "Yes" ( $n=75,63 \%)$. This was followed by "I don't know" ( $n=28,24 \%$ ) and "No" ( $n=16$, $13 \%)$ as shown below in Figure 13.

No single professional is capable of knowing everything about all of the critical issues confronting educational leaders on a daily basis. Further, even if that leader has had training in the past, she or he cannot be expected to remember all the information that could help in making a quality, timely decision. One possible solution is the development of a screening tool that can help educators more effectively identify students who may have FASD. An FASD-specific screening tool utilized within school settings could be extremely helpful for educational professionals by helping guide and inform decision-making with greater confidence.

\section{Discussion}

Response rate from the survey was strong at $37 \%$ with of ratio of nearly 4 male participants ( $n=93,79 \%$ ) to each female participant $(n=25,21 \%)$. This ratio tends to indicate there are substantially more males serving as superintendents in the state of Minnesota. Additionally, the greater majority ( $n=107,90 \%$ ) of respondents had held their administrative license for 5 or more years.

\section{FASD diagnostic knowledge}

FASD is often considered an invisible disorder because those impacted rarely present physical indicators that would betray their condition(s). Even if deformities are present at birth, children tend to outgrow them as they mature making visible identification more challenging $[25,26]$. Unfortunately, only about $30 \%$ of respondents recognized that children with Fetal Alcohol Spectrum Disorder typically do not have physical deformities such wide-set eyes, thin upper lip, smooth philtrum, upturned nose, epicanthal folds, or a reduced head size. Until such perceptions are changed, FASD will likely persist as an under-identified disorder.

This missed or inaccurate diagnosis of FASD may contribute to the underestimation of criminal justice-in- volvement of individuals with FASD. In this study, only $44 \%$ believed that at most $40 \%$ of those with FASD would enter the criminal justice system in some capacity. In contrast, research has found that the actual prevalence rate of criminal justice-involvement by individuals with FASD may exceed 60\% [15]. However, it was noteworthy that a second group consisting of $31 \%$ of the sample estimated that $61-80 \%$ of individuals with FASD would become involved in the criminal justice system. This gap in estimates between these two groups indicates an area appropriate for increased funding, research, and training.

\section{Training}

Training helps increase awareness and knowledge of FASD. It also assists in increasing the effectiveness of district resource allocation at the highest levels of district administration. Over half of the 120 respondents 61\%) reported never receiving any training on the neurobehavioral impairments experienced by individuals with FASD. For the minority of respondents that received some form of training, the training they had received typically took place over 5 years ago. With a gap in training of at least 5 years, the science and understanding in the field may have dramatically shifted. To increase the efficacy of training, it must remain timely and relevant.

Among respondents who had had some training, almost $90 \%(n=41)$ recalled the educational developmental impairments of FASD in school age children. Similarly, the vast majority of respondents (75\%) recalled behavioral developmental impairments of FASD in school age children. In contrast, no one $(0 \%)$ could recall longterm planning for students with a FASD. Similarly, very few could recall training content on legal and policy issues (1\%), clinical background (2\%), or identification and treatment ( $\mathrm{n}=2,2 \%$ ) on FASD. These disparities point to possible areas for improvement in the training provided to school district staff.

Despite the promise of improved trainings, such programs would not be a panacea. Although most respondents believe that a continuing education course on the neurobehavioral impairments of FASD would be helpful $(70 \%)$, a sizable minority $(27 \%)$ were uncertain that such training would be helpful. Similar results were observed when respondents were asked about the potential use of a FASD screening tool in daily practice. These results, although not initially intuitive, could be due to the severe lack of timely and relevant training to make the information both actionable and valuable along with other logistical and practical time constraints.

\section{Professional experience with FASD}

Based on this survey, just over half (52\%) of Minnesota superintendents appear to have been personally involved with significant behavioral concerns of FASD students. This striking figure reinforces the perception that FASD behavioral cases are rising to the highest lev- 
els of district administration throughout the state. As a result, superintendents overwhelmingly agree that continuing education (70\%) and availability of a FASD screening tool (63\%) would be beneficial in handling such cases.

An informed, collaborative approach to understanding and intervening will help educators and families meet the complex needs of students with FASD. Providers from areas of mental health, social services, developmental disabilities, and law enforcement allow for provision of interventions that draw upon expertise within the schools and surrounding community. Early diagnosis, when paired with intensive and appropriate intervention, has the potential to positively impact the life of an individual with FASD.

\section{Limitations}

A key limitation of this study was the response rate. Specifically, only 120 out of 325 (36.9\%) potential participants responded to the survey. Although the findings are still informative in an area with a dearth of research, this response rate could limit how well these findings generalize beyond this study. Further, this study only focused on superintendents in Minnesota. It is unclear how these findings might generalize to other states with more diverse populations or where FASD trainings may or may not be commonplace. Finally, this study only surveyed superintendents. The inclusion of other education professionals such as teachers could alter findings. This is important as teachers are more likely to have prolonged interactions with students than administrators. Nonetheless, these findings are an important first step in better understanding the familiarity of education professionals with FASD. In turn, these findings emphasize the importance for future research on a grander scale.

\section{Suggestions for future research}

The current study demonstrates the potential for expansion in several ways. First, this study should be replicated in more diverse samples. Foremost, replicating this study in a national sample could be helpful in understanding how generalizable the findings are to other regions and cultures. Further, surveying other professionals beyond superintendents could prove fruitful. Specifically, surveying other education professionals (e.g., principals, special education directors, school counselors, and teachers), mental health (e.g., school social workers and school psychologists), and criminal justice (e.g., school resource officers) may provide previously undiscovered insights into the understanding of FASD. For example, school resource officers could help illuminate the initial criminal justice system contacts of adolescents with FASD. Alternatively, applying the survey to caregivers, immediate family members, relatives, and the individuals themselves also may yield interesting results. Second, expansion of these surveys to gather information on different educational approaches and techniques used in this population could be essential in the identification of evidence based practices and best practices in this area. For example, survey results may be informative in gauging the utility of employing trauma-informed care approaches in students with FASD. Third, the findings from such survey work can be used to inform the development and refinement of FASD-related training programs for educational and other professionals. Such endeavors could explore if ND-PAE criteria can alleviate issues related to missed and inaccurate diagnosis of FASD. As laid out in this section, a systematic and nuanced approach to exploring the varied understanding of FASD has the potential to improve outcomes for those with the disorder.

\section{Conclusion}

Because at least $2 \%$ to $5 \%$ of the U.S. population suffers from FASD [1], educators can make a big difference in improving the identification of this often unrecognized disorder. In particular, school district administrators have the potential to provide critical and timely advocacy for valuable training, assistance, and prevention on behalf of students with FASD. However, limitations in knowledge in the area of FASD make such advocacy unlikely. These areas are critical to address in educational institutions as knowledge of the disorder is essential in taking the first steps to preventing the short- and longterm consequences of FASD.

As highlighted by the findings of this survey, many superintendents lack a firm grasp on the educational and developmental impairments of students with FASD. Although school district superintendents acknowledged that there are educational developmental impairments in school age children with FASD, very few could recall key pieces of knowledge relevant to FASD. This includes the screening and assessment, clinical background, treatment, and legal and policy issues of FASD. For example, only about $30 \%$ of respondents recognized that the vast majority of students with FASD lack physical indicators of the disorder. To help improve short- and long-term outcomes for students with FASD, there is a need for advanced FASD training for educational professionals.

Superintendents are an important target as these professionals are likely to come into contact with students with FASD due to the disorder's educational and behavioral impacts. These impairments may necessitate removal from classroom settings and require consideration of more severe penalties such as long-term suspension and expulsion. If superintendents were more familiar with FASD, these important decision points could serve as an opportunity for advocacy. For example, referrals for assessment and the development of case management plans could prevent permanent removal from school or criminal justice involvement. To address current shortcomings in the field, research is needed to explore everything from how well educators and other professionals understand FASD to identifying 
ways to improve the screening and assessment of FASD in education settings. If improvements are not made, trouble in school settings experienced by students with FASD may increase the likelihood of worse outcomes including involvement in the juvenile and adult criminal justice systems.

\section{References}

1. May PA, Gossage JP, Kalberg WO, Robinson LK, Buckley D, et al. (2009) Prevalence and epidemiologic characteristics of FASD from various research methods with an emphasis on recent in-school studies. Dev Disabil Res Rev 15: 176-192.

2. Clarke ME, Gibbard WB (2003) Overview of fetal alcohol spectrum disorders for mental health professionals. Can Child Adolesc Psychiatr Rev 12: 57-63.

3. Fast DK, Conry J (2009) Fetal alcohol spectrum disorders and the criminal justice system. Dev Disabil Res Rev 15: 250-257.

4. Norman AL, O'Brien JW, Spadoni AD, Tapert SF, Jones $\mathrm{KL}$, et al. (2013) A functional magnetic resonance imaging study of spatial working memory in children with prenatal alcohol exposure: contribution of familial history of alcohol use disorders. Alcohol Clin Exp Res 37: 132-140.

5. Nunez CC, Roussotte F, Sowell ER (2011) Focus on: structural and functional brain abnormalities in fetal alcohol spectrum disorders. Alcohol Res Health 34: 121-131.

6. Rasmussen C (2005) Executive functioning and working memory in fetal alcohol spectrum disorder. Alcohol Clin Exp Res 29: 1359-1367.

7. Kalberg WO, Buckley D (2007) FASD: What types of intervention and rehabilitation are useful? Neurosci Biobehav Rev 31: 278-285.

8. Streissguth AP, Barr HM, Sampson PD, Bookstein FL (1994) Prenatal alcohol and off spring development: the first fourteen years. Drug Alcohol Depend 36: 89-99.

9. Wheeler SM, Stevens SA, Sheard ED, Rovet JF (2012) Facial memory deficits in children with fetal alcohol spectrum disorders. Child Neuropsychol 18: 339-346.

10. Kodituwakku PW, Handmaker NS, Cutler SK, Weathersby EK, Handmaker SD (1995) Specific impairments in self-regulation in children exposed to alcohol prenatally. Alcohol Clin Exp Res 19: 1558-1564.

11. Bower E, Szajer J, Mattson SN, Riley EP, Murphy C (2013) Impaired odor identification in children with histories of heavy prenatal alcohol exposure. Alcohol 47: 275-278.

12. Mattson SN, Crocker N, Nguyen TT (2011) Fetal alcohol spectrum disorders: neuropsychological and behavioral features. Neuropsychol Rev 21: 81-101.

13. Carolyn Blackburn, Barry Carpenter, Jo Egerton (2010)
Shaping the future for children with foetal alcohol spectrum disorders. Support for Learning 25: 139-145.

14. Streissguth AP, Randels SP, Smith DF (1991) A test-retest study of intelligence in patients with fetal alcohol syndrome: implications for care. Journal of the American Academy of Child \& Adolescent Psychiatry 30: 584-587.

15. Streissguth AP, Barr HM, Kogan J, Bookstein FL (1996) Understanding the occurrence of secondary disabilities in clients with fetal alcohol syndrome and fetal alcohol effects. Final report to the Centers for Disease Control and Prevention (CDC), Seattle, University of Washington, WA.

16. Famy C, Streissguth AP, Unis AS (1998) Mental illness in adults with fetal alcohol syndrome or fetal alcohol effects. American Journal of Psychiatry 155: 552-554.

17. Green CR, Mihic AM, Nikkel SM, Stade BC, Rasmussen C, et al. (2009) Executive function deficits in children with fetal alcohol spectrum disorders (FASD) measured using the Cambridge Neuropsychological Tests Automated Battery (CANTAB). J Child Psychol Psychiatry 50: 688-697.

18. Hon Susan Shepard Carlson, Hon Susan Shepard Carlson (2011) A judicial perspective on issues impacting the trial courts related to Fetal Alcohol Spectrum Disorders. The Journal of Psychiatry \& Law 39: 73-119.

19. Edmonds K, Crichton S (2008) Finding ways to teach students with FASD, a research study. International Journal Of Special Education 23: 54-73.

20. Susan Ryan, Dianne L Ferguson (2006) On, yet under the radar: Students with Fetal Alcohol Syndrome Disorder. Exceptional Children 72: 363-379.

21. Thiel K, Baladerian N, Boyce K, Cantos O, Davis L, et al. (2010) Fetal Alcohol Spectrum Disorders and victimization: Implications for families, educators, social services, law enforcement, and the judicial system. Journal of Psychiatry \& Law 39: 121-157.

22. Gunn J (2013) Meeting the needs of children with foetal alcohol spectrum disorder through research based interventions. New Zealand Journal of Teachers' Work 10: 148-168.

23. Olson H, Oti R, Gelo J, Beck S (2009) "Family matters": Fetal alcohol spectrum disorders and the family. Dev Disabil Res Rev 15: 235-249.

24. IBM Corp. Released 2013. IBM SPSS Statistics for Windows, Version 22.0. Armonk, NY: IBM Corp. Alcoholism: Clinical and Experimental Research 19: 1558-1564.

25. Murawski NJ, Moore EM, Thomas JD, Riley EP (2015) Advances in diagnosis and treatment of Fetal Alcohol Spectrum Disorders: From Animal Models to Human Studies. Alcohol Res 37: 97-108.

26. Spohr HL, Willms J, Steinhausen HC (1994) The fetal alcohol syndrome in adolescence. Acta Paediatr Suppl 404: 19-26. 